\title{
Teichmüller spaces of non-discrete groups
}

\author{
Jun Hu* and Francisco G. Jimenez-Lopez ${ }^{\dagger}$
}

\begin{abstract}
The concept of Teichmüller space of a Fuchsian group can be extended to any non-discrete group of conformal homeomorphisms of the hyperbolic plane. In this paper, we first present three models of Teichmüller space fulfilling that goal. Then we use them to study the Teichmüller spaces $T(G)$ of the non-discrete subgroups $G$ of $\operatorname{PSL}(2, \mathbb{R})$. We show that $T(G)$ is not trivial if and only if $G$ is a subgroup consisting of hyperbolic elements with two common fixed points and accumulating to at least one non-identity element. Furthermore, we show that if $T(G)$ is not trivial, then (1) $T(G)$ is conformally equivalent to the open unit disk, (2) the Teichmüller metric on $T(G)$ is equal to the hyperbolic metric on the disk, and (3) the length spectrum is just a pseudometric on $T(G)$ and when restricted to a one-dimensional real slice, it is a metric coinciding with the Teichmüller metric.
\end{abstract}

\section{Ei-diskreettien ryhmien Teichmüllerin avaruudet}

Tiivistelmä. Fuchsin ryhmän Teichmüllerin avaruuden käsite voidaan yleistää mihin tahansa hyperbolisen tason konformisten homeomorfismien ei-diskreettiin ryhmään. Tässä työssä esittelemme aluksi kolme Teichmüllerin avaruuden mallia, jotka toteuttavat tämän tavoitteen. Sitten käytämme niitä ryhmän $\operatorname{PSL}(2, \mathbb{R})$ ei-diskreettien aliryhmien $G$ Teichmüllerin avaruuksien $T(G)$ tutkimiseen. Osoitamme, että $T(G)$ on epätriviaali, jos ja vain jos aliryhmä $G$ sisältää hyperbolisia alkioita, joilla on kaksi yhteistä kiintopistettä ja jotka kasaantuvat ainakin yhtä ei-yksikköalkiota kohti. Lisäksi osoitamme, että jos $T(G)$ on epätriviaali, niin (1) $T(G)$ on konformisessa vastaavuudessa yksikkökiekon kanssa, (2) avaruuden $T(G)$ Teichmüllerin metriikka on sama kuin kiekon hyperbolinen metriikka, ja (3) pituusspektri on avaruuden $T(G)$ pseudometriikka, joka yksiulotteiseen reaaliseen viipaleeseen rajoitettuna yhtyy Teichmüllerin metriikkaan.

\section{Introduction}

In this section, we provide background and give the statements of the results. Denote by $\mathbb{C}$ the complex plane and $\hat{\mathbb{C}}$ the extended complex plane (the Riemann sphere). Let $\mathbb{H}$ be the upper half plane and $\mathbb{H}^{-}$the lower half plane, and let $\mathbb{R}$ be the real line and $\hat{\mathbb{R}}$ the extended real line. Given a complex-valued measurable function $\mu$ defined on $\mathbb{H}$ with norm $\|\mu\|_{\infty}<1$, denote by

$$
f^{\mu}: \hat{\mathbb{C}} \rightarrow \hat{\mathbb{C}}
$$

the unique quasiconformal mapping fixing 1,0 and $\infty$ with complex dilatation $\frac{\bar{\partial} f^{\mu}}{\partial f^{\mu}}$ equal to $\mu$ on $\mathbb{H}$ and $\overline{\mu(\bar{z})}$ for any $z \in \mathbb{H}^{-}$, and denote by

$$
f_{\mu}: \hat{\mathbb{C}} \rightarrow \hat{\mathbb{C}}
$$

https://doi.org/10.54330/afm.114460

2020 Mathematics Subject Classification: Primary 30F60; Secondary 30F99.

Key words: Teichmüller space, length spectrum metric, non-discrete groups.

* Research partially supported by PSC-CUNY research awards, a CUNY fellowship leave grant, and a visiting professorship at NYU Shanghai.

${ }^{\dagger}$ Research partially supported by CONACYT posdoctoral fellowship.

(c) 2022 The Finnish Mathematical Society 
the unique quasiconformal mapping fixing 1, 0 and $\infty$ with complex dilatation equal to $\mu$ on $\mathbb{H}$ and 0 on $\mathbb{H}^{-}$.

Clearly, each mapping $f^{\mu}$ preserves $\mathbb{H}$ and hence $\mathbb{R}$. Define by

$$
\mathbb{H P}(\mathbb{H} \text { preserving })=\left\{f^{\mu}:\|\mu\|_{\infty}<1\right\}
$$

and

$$
\text { LC (lower conformal })=\left\{f_{\mu}:\|\mu\|_{\infty}<1\right\} .
$$

Recall that the Schwarzian derivative of a conformal mapping $f: A \subseteq \hat{\mathbb{C}} \rightarrow \hat{\mathbb{C}}$ is given by

$$
S_{f}=\left(\frac{f^{\prime \prime}}{f^{\prime}}\right)^{\prime}-\frac{1}{2}\left(\frac{f^{\prime \prime}}{f^{\prime}}\right)^{2} .
$$

In the following, we first recall three different models that are used to define/represent the universal Teichmüller space $T(1)$.

Definition 1.1. (Using quasisymmetric homeomorphisms of $\hat{\mathbb{R}}$ )

$$
T(1)=\mathbb{H P} / \sim,
$$

where

$$
\left.f^{\mu_{1}} \sim f^{\mu_{2}} \Longleftrightarrow f^{\mu_{1}}\right|_{\mathbb{R}}=\left.f^{\mu_{2}}\right|_{\mathbb{R}} .
$$

Definition 1.2. (Using quasidisks)

$$
T(1)=\mathrm{LC} / \sim,
$$

where

$$
\left.f_{\mu_{1}} \sim f_{\mu_{2}} \Leftrightarrow f_{\mu_{1}}\right|_{\mathbb{H}^{-}}=\left.f_{\mu_{2}}\right|_{\mathbb{H}^{-}} \Longleftrightarrow S_{\left.f_{\mu_{2}}\right|_{\mathbb{H}^{-}}}=S_{\left.f_{\mu_{2}}\right|_{\mathbb{H}^{-}}} .
$$

Definition 1.3. (Using Bers' embedding)

$$
T(1)=\left\{S_{\left.f_{\mu}\right|_{\mathbb{H}^{-}}}: f_{\mu} \in \mathrm{LC}\right\} .
$$

Let $G \subseteq \operatorname{PSL}(2, \mathbb{R})$ be a Fuchsian group, i.e., a discrete group of Möbius transformations preserving $\mathbb{H}$. Corresponding to three different representations of the universal Teichmüller space $T(1)$, the Teichmüller space $T(G)$ of $G$ can be defined by using one of the following three models:

Definition 1.4. (Using quasisymmetric homeomorphisms of $\hat{\mathbb{R}}$ )

$$
T(G)=\left\{f^{\mu} \in \mathbb{H P}: f^{\mu} \circ g \circ\left(f^{\mu}\right)^{-1} \in \operatorname{PSL}(2, \mathbb{R})(\forall g \in G)\right\} / \sim,
$$

where

$$
\left.f^{\mu_{1}} \sim f^{\mu_{2}} \Longleftrightarrow f^{\mu_{1}}\right|_{\mathbb{R}}=\left.f^{\mu_{2}}\right|_{\mathbb{R}} .
$$

Definition 1.5. (Using quasidisks)

$$
T(G)=\left\{f_{\mu} \in \mathrm{LC}: f_{\mu} \circ g \circ f_{\mu}^{-1} \in \operatorname{PSL}(2, \mathbb{C})(\forall g \in G)\right\} / \sim,
$$

where

$$
\left.f_{\mu_{1}} \sim f_{\mu_{2}} \Longleftrightarrow f_{\mu_{1}}\right|_{\mathbb{H}^{-}}=\left.f_{\mu_{2}}\right|_{\mathbb{H}^{-}} .
$$

Definition 1.6. (Using Bers' embedding)

$$
T(G)=\left\{\phi=S_{\left.f_{\mu}\right|_{\mathbb{H}^{-}}}: f_{\mu} \in \mathrm{LC} \text { and } \phi(g(z))\left[g^{\prime}(z)\right]^{2}=\phi(z)(\forall g \in G)\right\} .
$$

Clearly, all of the previous definitions of Teichmüller spaces of Fuchsian groups can be directly copied to define Teichmüller spaces of non-discrete groups of conformal homeomorphisms of the upper half plane. We say that $G$ is elementary if there exists a finite $G$-orbit (see the precise definition in the next section just before Theorem 2.3). The first result of this paper is the following. 
Theorem 1. Assume that $G$ is a non-discrete subgroup of $\operatorname{PSL}(2, \mathbb{R})$.

(1) If $G$ is non-elementary, then $T(G)$ is trivial.

(2) If $G$ is elementary, $T(G)$ is not trivial if and only if $G$ consists of hyperbolic elements with common fixed points (with multipliers having nonzero finite accumulation points). Furthermore, if $T(G)$ is not trivial, then $T(G)$ is conformally equivalent to the unit disk and hence is a complex Banach manifold with one chart map.

The Teichmüller metric is well defined on $T(G)$; that is, given any two points $\left[f^{\mu_{1}}\right]$ and $\left[f^{\mu_{2}}\right]$ of $T(G)$,

$$
d_{T}\left(\left[f^{\mu_{1}}\right],\left[f^{\mu_{2}}\right]\right)=\log \inf K(f),
$$

where $f=f^{\mu_{1}} \circ\left(f^{\mu_{2}}\right)^{-1}, K(f)$ denotes the maximal dilatation of $f$, and the infimum is taken over all representatives of $\left[f^{\mu_{1}}\right]$ and $\left[f^{\mu_{2}}\right]$.

Now we consider another metric that can also be defined on the nontrivial case of $T(G)$ when $G$ is a non-discrete subgroup of $\operatorname{PSL}(2, \mathbb{R})$. Let us recall how the length spectrum is defined on the Teichmüller space $T\left(S_{0}\right)$ of a Riemann surface $S_{0}$.

Let $S_{0}$ be a Riemann surface. A marked Riemann surface is a pair $(S, f)$ with $f: S_{0} \rightarrow S$ being a quasiconformal mapping. Two pairs $\left(S_{1}, f_{1}\right)$ and $\left(S_{2}, f_{2}\right)$ are equivalent if there exists a conformal mapping $c: S_{1} \rightarrow S_{2}$ such that $c \circ f_{1}$ is homotopic to $f_{2}$. The Teichmüller space $T\left(S_{0}\right)$ is the set of equivalence classes $[S, f]$. A simple closed curve on $S$ is said to be essential if it is neither homotopic to a point nor to a puncture and nor to a boundary component. Let $\Sigma_{S}$ be the collection of simple closed curves on $S$ containing one and exactly one representative from each homotopy class of essential curves. For each $\gamma \in \Sigma_{S}$, let $l_{S}(\gamma)$ denote the length of the shortest curve in the homotopy class of $\gamma$ under the metric on $S$. The length spectrum is defined by

$$
d_{L}\left(\left[S_{1}, f_{1}\right],\left[S_{2}, f_{2}\right]\right)=\log \sup _{\gamma \in \Sigma_{S_{1}}}\left\{\left(\frac{l_{S_{2}}\left(f_{2} \circ f_{1}^{-1}(\gamma)\right)}{l_{S_{1}}(\gamma)}\right)^{ \pm 1}\right\} .
$$

The length spectrum is a pseudometric on $T\left(S_{0}\right)$. In many cases, it is a metric. It was proved in [9] by Sorvali that $d_{L}$ is bi-Lipschitz to $d_{T}$ on the Teichmüller space of a torus. Later Li proved in [5] that the two metrics $d_{L}$ and $d_{T}$ define the same topology on the Teichmüller space of a compact Riemann surface, and he proved in [6] that the two metrics are not bi-Lipschitz if the genus of the Riemann surface is bigger than 1. Then Liu [7] proved the topological equivalence of the two metrics on the Teichmüller spaces of hyperbolic surfaces with boundary and of finite type. In Liu's paper, the reduced Teichmüller space is considered, since $d_{L}$ does not define a metric on every marked Teichmüller space of surfaces with boundary (see [3]). Shiga [8] showed that Liu's result fails in general on Teichmüller spaces of Riemann surfaces of infinite type and he also introduced a sufficient condition for Liu's result to hold on such Teichmüller spaces.

Except for the torus case, one usually considers Teichmüller spaces of hyperbolic Riemann surfaces. In fact, if $S_{0}$ is a hyperbolic Riemann surface, then the length spectrum on $T\left(S_{0}\right)$ can be alternatively defined as follows. Let $G$ be a subgroup of $\operatorname{PSL}(2, \mathbb{R})$ representing a hyperbolic Riemann surface $S_{0}$; that is, $S_{0}=\mathbb{H} / G$. For any hyperbolic transformation $g \in G$, let $\tau(g)$ denote the multiplier of $g$. Then

$$
\left.d_{L}\left(\left[f^{\mu_{1}}\right],\left[f^{\mu_{2}}\right]\right)\right)=\log \sup _{g \in G, g \text { hyperbolic }}\left\{\left|\frac{\log \tau\left(f^{\mu_{1}} \circ g \circ\left(f^{\mu_{1}}\right)^{-1}\right)}{\log \tau\left(f^{\mu_{2}} \circ g \circ\left(f^{\mu_{2}}\right)^{-1}\right)}\right|^{ \pm 1}\right\} .
$$


One can see clearly that this alternative definition of $d_{L}$ extends to the nontrivial case of $T(G)$ when $G$ is a non-discrete subgroup of $\operatorname{PSL}(2, \mathbb{R})$. In this paper, we also prove the following theorem.

Theorem 2. Let $G$ be a non-discrete subgroup of $P S L(2, \mathbb{R})$ consisting of only hyperbolic elements with two common fixed points. Then

(1) the Teichmüller metric on $T(G)$ is equal to the hyperbolic metric on the disk under the identification map in Theorem 1 , and

(2) the length spectrum is just a pseudometric on $T(G)$ and when restricted to the horizontal diameter of the disk (under the identification map), it is a metric coinciding with the Teichmüller metric.

In the following two sections, we prove the two theorems with quite elementary and self-contained approaches.

Acknowledgement. This work was inspired by a talk given by Professor Clifford Earle at CUNY Graduate Center quite a few years ago. Both authors hope that this paper honors his memory. They also wish to thank the referees for their comments and corrections of typos.

\section{Proof of Theorem 1}

Let $G$ be a non-discrete subgroup of $\operatorname{PSL}(2, \mathbb{R})$. Bers' embedding (see Definition 1.6) is an injection from $T(G)$ into the space of holomorphic maps, which is a complex linear space. Using Definition 1.6, one can also obtain an estimate on the dimension of $T(G)$.

Theorem 2.1. Let $G$ be a non-discrete subgroup of $\operatorname{PSL}(2, \mathbb{R})$. Then the (complex) dimension of $T(G)$ is at most 1.

Proof. We use Definition 1.6 to define $T(G)$. Then given any two non-trivial elements $\phi, \varphi \in T(G)$,

$$
\frac{\phi(g(z))}{\varphi(g(z))}=\frac{\phi(z)}{\varphi(z)}
$$

for any $g \in G$. Since $G$ is not discrete, there exists a sequence $\left\{g_{n}\right\}_{n=1}^{\infty}$ of elements in $G$ converging to the identity element. Therefore, $\frac{\phi(z)}{\varphi(z)}$ is equal to a constant $c$ and then $\phi=c \varphi$. This means that $\operatorname{dim}_{\mathbb{C}} T(G) \leq 1$.

Now we prove a proposition due to Earle.

Proposition 2.2. (Earle) Let $G$ be a non-discrete subgroup of $\operatorname{PSL}(2, \mathbb{R})$.

(1) If $G$ contains infinitely many elliptic elements fixing a common point $z_{0} \in \mathbb{H}^{-}$, then $T(G)$ is trivial, i.e., a single-point space.

(2) If $G$ contains infinitely many parabolic elements fixing a common point $x_{0} \in \hat{\mathbb{R}}$ and with their translation amounts accumulating at finite nonzero values, then $T(G)$ is trivial.

(3) If $G$ only contains hyperbolic elements fixing two common points $x_{1}, x_{2} \in$ $\hat{\mathbb{R}}$, then $T(G)$ is nontrivial and under Bers' embedding, it is conformally equivalent to the unit open disk $\mathbb{D}$.

Let $A$ be a simply connected domain conformally equivalent to the unit disk with Poincaré density $\eta$. Given a holomorphic map $\varphi: A \rightarrow \mathbb{C}$, define

$$
\|\varphi\|_{A}=\sup _{z \in A}|\varphi(z)| \eta(z)^{-2} \text {. }
$$


It is well-known that $\|\varphi\|_{A} \leq 6$ (see [4] for a proof).

Proof. (1) We take the unit disk $\mathbb{D}$ as a model for the hyperbolic plane. We may assume that $z_{0}=0$ and $G$ contains elements of the form $g_{t_{k}}(z)=e^{i t_{k}} z$, where $t_{k} \in \mathbb{R}$ and $\left\{e^{i t_{k}}\right\}_{k=1}^{\infty}$ has accumulation points on the unit circle. Let $f_{\mu}$ represent a point of $T(G)$ in Definition 1.5. In this setting, we let $f_{\mu}$ be conformal on $\mathbb{D}$. Let $\varphi=S_{f_{\mu} \mid \mathbb{D}}$. Then for any $t_{k} \in \mathbb{R}$,

$$
\varphi(z)=\varphi\left(g_{t_{k}}(z)\right)\left[g_{t_{k}}^{\prime}(z)\right]^{2}=\varphi\left(e^{i t_{k}} z\right) e^{2 i t_{k}} .
$$

Therefore, the function $F(z)=\varphi(z) z^{2}$ satisfies $F(z)=F\left(e^{i t_{k}} z\right)$ for all $t_{k} \in \mathbb{R}$. Since $\left\{e^{i t_{k}}\right\}_{k=1}^{\infty}$ has accumulation points on the unit circle, it follows that $F$ is a constant, say $F \equiv C$, and hence

Since

$$
\varphi(z)=\frac{C}{z^{2}}
$$

$$
\|\varphi\|_{\mathbb{D}}=\sup _{z \in \mathbb{D}}\left|\frac{C}{z^{2}}\right|\left(1-|z|^{2}\right) \leq 6,
$$

the constant $C$ must be 0 and then the first part of Proposition 2.2 follows.

(2) We may assume, without loss of generality, that $x_{0}=\infty$. Then $G$ contains elements of the form $g_{t_{k}}(z)=z+t_{k}$, where $t_{k} \in \mathbb{R}$ and $\left\{t_{k}\right\}_{k=1}^{\infty}$ has nonzero finite accumulation points. Let $\left[f_{\mu}\right]$ represent a point of $T(G)$ in Definition 1.5 and let $\varphi=S_{\left.f_{\mu}\right|_{\mathbb{H}^{-}}}$. Then for any $t \in \mathbb{R}$

$$
\varphi(z)=\varphi\left(g_{t_{k}}(z)\right)\left[g_{t_{k}}^{\prime}(z)\right]^{2}=\varphi\left(z+t_{k}\right)
$$

It follows that $\varphi$ is constant, say $\varphi \equiv C$. Since

$$
\|\varphi\|_{\mathbb{H}^{-}}=\sup _{z \in \mathbb{H}^{-}}|C| 4(\operatorname{Im}(z))^{2} \leq 6
$$

the constant $C$ must be 0 and then the second part of Proposition 2.2 follows.

(3) Let $G \subseteq \operatorname{PSL}(2, \mathbb{R})$ be a non-discrete group containing only hyperbolic elements with two common fixed points $x_{1}, x_{2} \in \hat{\mathbb{R}}$. Through conjugation by an element in $\operatorname{PSL}(2, \mathbb{R})$, we may assume that

$$
G=\left\{g_{t_{k}}(z)=e^{t_{k}} z: t \in \mathbb{R}\right\},
$$

where $t_{k} \in \mathbb{R}$ and $\left\{t_{k}\right\}_{k=0}^{\infty}$ has at least one nonzero finite accumulation point. Using Definition 1.6, one can see that $\varphi(z)=\frac{1}{z^{2}} \in T(G)$ and hence $\operatorname{dim}_{\mathbb{C}} T(G) \geq 1$. Combining this with Theorem 2.1, we see $\operatorname{dim}_{\mathbb{C}} T(G)=1$. The main work is to show that $T(G)$ is conformally identified with the unit open disk $\mathbb{D}$. To do this, we first prove that if Definition 1.6 is used to define $T(G)$, then $T(G)$ consists of the Schwarzian derivatives of all functions $f_{\beta}$ of the form

$$
f_{\beta}(z)= \begin{cases}z z^{\beta} & \text { if } z \in \mathbb{H}^{-}, \\ z \bar{z}^{\beta} & \text { if } z \in \mathbb{H},\end{cases}
$$

where $\beta$ is a constant with $|\beta|<1$. Note that each mapping $f_{\beta}$ is extremal in its class.

Let us first use Definition 1.5 for $T(G)$. Given $[f] \in T(G), f \circ g_{t_{k}} \circ f^{-1}$ is a Möbius transformation on $\hat{\mathbb{C}}$ fixing 0 and $\infty$. Thus

$$
\left(f \circ g_{t_{k}} \circ f^{-1}\right)(z)=\lambda_{t_{k}} z
$$

Moreover, since $f$ fixes 1 , we obtain

$$
f\left(e^{t_{k}}\right)=\left(f \circ g_{t_{k}} \circ f^{-1}\right)(1)=\lambda_{t_{k}} .
$$


Then for each $t_{k}$,

$$
f\left(e^{t_{k}}\right) f(z)=\lambda_{t_{k}} f(z)=\left(f \circ g_{t_{k}} \circ f^{-1}\right)(f(z))=f\left(e^{t_{k}} z\right) .
$$

Consider the universal covering map $p: \mathbb{C} \rightarrow \mathbb{C} \backslash\{0\}$ given by $p(\zeta)=e^{\zeta}$, and let $\tilde{f}: \mathbb{C} \rightarrow \mathbb{C}$ be the lift of $f: \mathbb{C} \backslash\{0\} \rightarrow \mathbb{C} \backslash\{0\}$ fixing 0 . For each $t_{k}$,

$$
e^{\tilde{f}\left(\zeta+t_{k}\right)}=f\left(e^{\zeta+t_{k}}\right)=f\left(e^{t_{k}}\right) f\left(e^{\zeta}\right)=e^{\tilde{f}\left(t_{k}\right)} e^{\tilde{f}(\zeta)} .
$$

This implies

$$
\tilde{f}\left(\zeta+t_{k}\right)=\tilde{f}(\zeta)+\tilde{f}\left(t_{k}\right)+c,
$$

where $c$ is constant. By letting $\zeta=0$ and using $\tilde{f}(0)=0$, we conclude $c=0$. Then

$$
\tilde{f}\left(\zeta+t_{k}\right)=\tilde{f}(\zeta)+\tilde{f}\left(t_{k}\right) \text { for each } t_{k}
$$

The strip $A=\{\zeta:-\pi<\operatorname{Im}(\zeta)<0\}$ is mapped by $p$ onto $\mathbb{H}^{-}$, where $f$ is holomorphic. Then $\tilde{f}$ is holomorphic on $A$ and for any $\zeta \in A$, we obtain

$$
\tilde{f}^{\prime}\left(\zeta+t_{k}\right)=\tilde{f}^{\prime}(\zeta)
$$

Since $\left\{t_{k}\right\}_{k=1}^{\infty}$ has finite accumulation points, it follows that $\tilde{f}^{\prime}$ is constant on $A$ and then $\tilde{f}(\zeta)=\alpha \zeta$ on $A$ for some $\alpha \in \mathbb{C} \backslash\{0\}$. Then

$$
f(z)=f\left(e^{\log z}\right)=e^{\tilde{f}(\log z)}=e^{\alpha \log z}=z^{\alpha} \text { for all } z \in \mathbb{H}^{-},
$$

where the branch of $\log$ is given by

$$
\log z=\log |z|+i \arg z \text { for }-\pi<\arg z<\pi .
$$

The region $\tilde{f}(A)$ is a strip bounded by the lines $l_{1}=\{z: z=\alpha x$ for $x \in \mathbb{R}\}$ and $l_{2}=\{z: z=\alpha(x-i \pi)$ for $x \in \mathbb{R}\}$. Let $\alpha=a+b i$. Since no two points in the closure of $\tilde{f}(A)$ can project to the same point under $p$, we must have $a \neq 0$. Moreover, $l_{2}$ intersects the $y$-axis at $-\frac{\left(b^{2}+a^{2}\right) \pi}{a} i$ and $l_{1}$ intersects the $y$-axis at the origin. It follows that the line segment between the origin and $-\frac{\left(b^{2}+a^{2}\right) \pi}{a} i$ is contained in $\tilde{f}(A)$. Then

$$
\frac{\left(b^{2}+a^{2}\right) \pi}{a}<2 \pi
$$

that is,

$$
(a-1)^{2}+b^{2}<1 .
$$

Thus, $\alpha=a+b i=1+\beta$ with $|\beta|<1$. We conclude that

$$
f(z)=z^{\alpha}=z^{1+\beta}=z z^{\beta} \text { for } z \in \mathbb{H} \text {. }
$$

Now let $z_{1}=x+\pi i$ and $z_{2}=x-\pi i$, where $x \in \mathbb{R}$. These points are mapped by $p$ to the same point on $\mathbb{C} \backslash\{0\}$. Since $p\left(\tilde{f}\left(z_{1}\right)\right)=f\left(p\left(z_{1}\right)\right)=f\left(p\left(z_{2}\right)\right)=p\left(\tilde{f}\left(z_{2}\right)\right)$, it follows that $\tilde{f}\left(z_{1}\right)=\tilde{f}\left(z_{2}\right)+2 k \pi i$, where $k$ is an integer. Since $\tilde{f}: \mathbb{C} \rightarrow \mathbb{C}$ is the lift of $f: \mathbb{C} \backslash\{0\} \rightarrow \mathbb{C} \backslash\{0\}$ fixing 0 , it follows that $k=1$. Then

$$
\tilde{f}(x+\pi i)=\tilde{f}(x-\pi i)+2 \pi i=\alpha(x-\pi i)+2 \pi i=(x+\pi i)+\beta(x-\pi i) .
$$

This verifies that if $z_{1}=x+\pi i$, then $\tilde{f}\left(z_{1}\right)=z_{1}+\beta \overline{z_{1}}$ for any $x \in \mathbb{R}$.

Let $B=\{\zeta: 0<\operatorname{Im}(\zeta)<\pi\}$. Now we define an extension $\tilde{f}_{\beta}$ of $\left.\tilde{f}\right|_{A}$ to the closure of the union $A \cup B$ by

$$
\tilde{f}_{\beta}(z)= \begin{cases}\alpha z & \text { if } z \in A \\ z+\beta \bar{z} & \text { if } z \in B .\end{cases}
$$


The Beltrami coefficient of $\tilde{f}_{\beta}$ is equal to the constant $\beta$. This map projects to a quasiconformal map $f_{\beta}$ from $\mathbb{C} \backslash\{0\}$ onto itself and hence it extends to a quasiconformal homeomorphism of $\mathbb{C}$ fixing the origin. (Note that the Beltrami coefficient of $f_{\beta}$ is equal to $\beta z / \bar{z}$ for each $z \in \mathbb{H}$. So if we use the notation $f_{\mu}$ given in the introduction, then $f_{\beta}$ should be denoted by $f_{\beta z / \bar{z}}$. Just for sake of notation, we denote it by $f_{\beta}$.) For any $z \in \mathbb{H}$,

$$
f_{\beta}(z)=f_{\beta}\left(e^{\log z}\right)=e^{\tilde{f}_{\beta}(\log z)}=e^{\log z+\mu \overline{\log z}}=z \bar{z}^{\beta} \text { for all } z \in \mathbb{H},
$$

where we use again the branch of log given by

$$
\log z=\log |z|+i \arg z \text { for }-\pi<\arg z<\pi .
$$

Hence $\tilde{f}_{\beta}$ projects to $f_{\beta}$ given by

$$
f_{\beta}(z)= \begin{cases}z z^{\beta} & \text { if } z \in \mathbb{H}^{-} \\ z \bar{z}^{\beta} & \text { if } z \in \mathbb{H} .\end{cases}
$$

Using Definition 1.5 for $T(G)$, one can see that $f_{\beta}$ represents the point $[f]$ in $T(G)$. In fact, it follows from Strebel's works [10] and [11] that $f_{\beta}$ is the unique extremal representation of $[f]$. Obviously, for two different values $\beta_{1}$ and $\beta_{2}$ in $\mathbb{D}$, $f_{\beta_{1}}$ and $f_{\beta_{2}}$ represent two different points in $T(G)$. Therefore, $T(G)$ can be identified with the open unit disk $\mathbb{D}$.

Furthermore, for any $\beta \in \mathbb{D}$,

$$
S_{\left.f_{\beta}\right|_{\mathbb{H}^{-}}}(z)=\frac{-\beta(\beta+2)}{2 z^{2}}
$$

Since the map $\beta \mapsto-\beta(\beta+2)$ is a holomorphic bijection between $\mathbb{D}$ and its image, it follows that $T(G)$ is conformally equivalent to $\mathbb{D}$ if Definition 1.6 is used to define $T(G)$. Furthermore, this conformal map enables us to view this Teichmüller space as a complex Banach manifold by using one chart map.

Using Proposition 2.2 and some background on the classification of non-discrete groups of Möbius transformations given in [2], we obtain the conclusions presented in our Theorem 1. In the following, we first summarize a minimal background on the classification and properties of non-discrete subgroups of $\operatorname{PSL}(2, \mathbb{R})$ that we need to apply in order to prove Theorem 1.

We identify the extended complex plane $\hat{\mathbb{C}}=\mathbb{C} \cup\{\infty\}$ with $\hat{\mathbb{R}}^{2}=\mathbb{R}^{2} \cup\{\infty\}$. In terms of quaternions, the three-dimensional hyperbolic space is expressed by

$$
\mathbb{H}^{3}=\{z+t j: z=x+y i \in \mathbb{C}, t>0\} .
$$

Then $\hat{\mathbb{C}}$ is the boundary of $\mathbb{H}^{3}$. The group $M\left(\hat{\mathbb{R}}^{2}\right)$ of orientation-preserving Möbius transforms of $\hat{\mathbb{R}}^{2}$ onto itself can be alternatively defined as the collection $\mathcal{M}$ of linear fractional maps (page 57, [2]); that is,

$$
M\left(\hat{\mathbb{R}}^{2}\right)=\mathcal{M}=\left\{g: g(z)=\frac{a z+b}{c z+d}, \text { where } a, b, c, d \in \mathbb{C} \text { and } a d-b c \neq 0\right\} .
$$

The group $\mathcal{M}$ acts as the group of directly conformal homeomorphisms of $\mathbb{H}^{3}$. A subgroup $G$ of $\mathcal{M}$ is said to be elementary if there exits a finite $G$-orbit in the closure of the hyperbolic space $\mathbb{H}^{3}$. Denote this finite $G$-orbit by $\left\{p_{1}, p_{2}, \cdots, p_{n}\right\}$. Elementary subgroups of $\mathcal{M}$ are classified at the beginning of Section 5.1 in [2]. 
Theorem 2.3. [2, page 84] Assume that a subgroup $G$ of $\mathcal{M}$ is elementary and let $\left\{p_{1}, p_{2}, \cdots, p_{n}\right\}$ be a finite $G$-orbit in the closure of $\mathbb{H}^{3}$. Then $G$ belongs to one of the following three types:

Type 1: Suppose that $n \geq 3$ or that $\left\{p_{1}, p_{2}, \cdots, p_{n}\right\}$ is not in $\hat{\mathbb{C}}$. Then $G$ contains only elliptic elements and the identity element.

Type 2: Suppose that $n=1$ and $p_{1} \in \hat{\mathbb{C}}$. Then $G$ is conjugate to a subgroup of $\mathcal{M}$, every element of which fixes $\infty$ and so is of the form $z \mapsto a z+b$, where $a \neq 0$.

Type 3: Suppose that $n=2$ and $p_{1}, p_{2} \in \hat{\mathbb{C}}$. Then $G$ is conjugate to a subgroup of $\mathcal{M}$, every element of which fixes $\{0, \infty\}$ and so is of the form $z \mapsto a z^{s}$, where $a \neq 0$ and $s^{2}=1$.

Note that all elements of $\operatorname{PSL}(2, \mathbb{R})$ preserve the hyperbolic plane $\mathbb{H}$. The following result is proved in [2].

Theorem 2.4. [2, Theorem 7.39.2] Let $g \in P S L(2, \mathbb{R})$ be elliptic with fixed point $v$ and angle of rotation $2 \theta$, where $0<\theta \leq \pi$. Let $h$ be any element of $\operatorname{PSL}(2, \mathbb{R})$ not fixing $v$. Then the commutator $g \circ h \circ g^{-1} \circ h^{-1}$ is hyperbolic.

The previous two theorems imply the following.

Corollary 2.5. If a subgroup $G$ of $\operatorname{PSL}(2, \mathbb{R})$ is elementary (as a subgroup of $\mathcal{M})$, then $G$ belongs to one of the following two types:

(1) $G$ consists of elliptic elements with a common axis of rotation and the identity element.

(2) $G$ consists of parabolic or hyperbolic elements sharing a common fixed point and the identity element.

Proof. It suffices to show that any elementary subgroup $G$ of $\operatorname{PSL}(2, \mathbb{R})$ can not be of type 3. Suppose such a subgroup $G$ exists. Then the finite orbit consists of two points $p_{1}$ and $p_{2}$. Through conjugation, we may assume that $p_{1}=0$ and $p_{2}=\infty$. Then all elements of $G$ must be of the form $z \mapsto a z^{s}$ with $a \neq 0$ and $s^{2}=1$. Since the elements of $G$ preserve the hyperbolic plane, it follows that $s \neq-1$ and all elements have the form $z \mapsto a z$ with $a \neq 0$. Thus, the finite $G$-orbit consists of only one point 0 or $\infty$. This is a contradiction. So $G$ can not be of type 3 .

As one can see, we are concerned with the nature of non-discrete subgroups $G$ of $\operatorname{PSL}(2, \mathbb{R}) \subset \mathcal{M}$. It remains to deal with the case when $G$ is non-discrete and non-elementary. The following two theorems are proved in Chapter 8 of [2].

Theorem 2.6. [2, Theorem 8.2.6] If a non-elementary group $G$ of isometries of $\mathbb{H}$ has no elliptic elements, then $G$ is discrete.

Theorem 2.7. [2, Theorem 8.4.1] A non-elementary group $G$ of isometries of $\mathbb{H}$ is discrete if and only if each elliptic element of $G$ has finite order (if exists).

Therefore, the following corollary holds.

Corollary 2.8. If a subgroup $G$ of $P S L(2, \mathbb{R})$ is non-elementary and non-discrete, then $G$ contains at least one elliptic element of infinite order.

Now we prove our Theorem 1.

Proof of Theorem 1. Let $G$ be a non-discrete subgroup of $\operatorname{PSL}(2, \mathbb{R})$. If $G$ is non-elementary, then by Corollary 2.8 we know that $G$ contains an elliptic element of infinite order. Thus, the first part of Proposition 2.2 implies that $T(G)$ is trivial. 
When $G$ is elementary, there are two cases to consider. If $G$ belongs to the first type of Corollary 2.5, then $G$ consists of elliptic elements with a common axis of rotation and the identity element. Because of the non-discreteness, $G$ has a sequence of elements accumulating to a rotation or the identity element. Hence $T(G)$ is trivial by applying the first part of Proposition 2.2.

If $G$ belongs to the second type of Corollary 2.5, then we need to do a little bit more work. We divide this case into two subcases. Without loss of generality, we assume the common fixed point is set at $\infty$.

Subcase 1: Assume that $G$ has a parabolic element. Then there exists a sequence of parabolic elements fixing $\infty$ and converging to a parabolic element or the identity element. Suppose not, then there exists a parabolic element $h(z)=z+b(b \neq 0)$ and a sequence of hyperbolic elements $\left\{g_{n}(z)=a_{n} z+b_{n}, a_{n} \neq 0,1\right\}$ accumulating to an element of $\operatorname{PSL}(2, \mathbb{R})$. Then $\left\{g_{n} \circ h \circ g_{n}^{-1}(z)=z-b_{n}+a_{n} b\right\}$ is a sequence of parabolic elements fixing $\infty$ and converging to an element of $\operatorname{PSL}(2, \mathbb{R})$. Therefore, $T(G)$ is trivial.

Subcase 2: Assume that $G$ contains only hyperbolic elements with one fixed point at $\infty$. We show that all hyperbolic elements of $G$ must share both fixed points. Suppose not, then there exists two elements $g_{1}(z)=a_{1} z+b_{1}$ and $g_{2}(z)=a_{2} z+b_{2}$, where $a_{1}, a_{2} \neq 0,1$ and $\frac{b_{1}}{1-a_{1}} \neq \frac{b_{2}}{1-a_{2}}$. It follows that $a_{1} b_{1}+b_{1}-a_{2} b_{1}-b_{2} \neq 0$ and then the commutator

$$
g_{1} \circ g_{2} \circ g_{1}^{-1} \circ g_{2}^{-1}(z)=z+\left(a_{1} b_{2}+b_{1}-a_{2} b_{1}-b_{2}\right)
$$

is a parabolic element. This is a contradiction. So all hyperbolic elements must share both fixed points. Hence, $T(G)$ is (conformally) identified with the open unit disk by applying the third part of Proposition 2.2.

In summary, $T(G)$ is not trivial if and only if $G$ consists of hyperbolic elements with two common fixed points (accumulating to a hyperbolic element or the identity) and the identity.

Remark 2.9. In fact, there is a different (long but interesting) method to handle the subcase 2 in the previous proof. We can show that if $T(G)$ is not trivial, then all hyperbolic elements of $G$ must share both fixed points. We use Definition 1.6 for $T(G)$. Given any $\varphi \in T(G)$,

$$
\varphi(g(z))\left[g^{\prime}(z)\right]^{2}=\varphi(z)
$$

for any $g \in G$. Let $g(z)=a z+b$ with $a \neq 0,1$. Suppose that $\varphi$ is not constantly equal to 0 . Around $\infty, \varphi(z)$ has a Maclaurin series expansion near $\infty$

$$
\varphi(z)=c_{0}+\frac{c_{1}}{z}+\frac{c_{2}}{z^{2}}+\frac{c_{3}}{z^{3}}+\cdots .
$$

Rewrite

$$
\frac{1}{g(z)}=\frac{1}{a z\left(1-\frac{b}{a z}\right)}=\frac{1}{a z}\left[1+\frac{b}{a z}+\left(\frac{b}{a z}\right)^{2}+\left(\frac{b}{a z}\right)^{3}+\cdots\right] .
$$

This series converges when $z$ is near $\infty$.

Substituting $\varphi$ and then $\frac{1}{g(z)}$ by their series expansions, we obtain

$$
\begin{aligned}
& c_{0}+\frac{c_{1}}{a z}\left(1+\frac{b}{a z}+\frac{b^{2}}{a^{2} z^{2}}+\frac{b^{3}}{a^{3} z^{3}}+\cdots\right)+\frac{c_{2}}{a^{2} z^{2}}\left(1+\frac{b}{a z}+\frac{b^{2}}{a^{2} z^{2}}+\frac{b^{3}}{a^{3} z^{3}}+\cdots\right)^{2}+\cdots \\
& =\frac{1}{a^{2}}\left[c_{0}+\frac{c_{1}}{z}+\frac{c_{2}}{z^{2}}+\frac{c_{3}}{z^{3}}+\cdots\right] .
\end{aligned}
$$


Note that $a \neq 0,1$. By checking the constant terms of both sides of the previous equation, we know $c_{0}=0$. Then by checking the coefficients of $\frac{1}{z}$, we know $c_{1}=0$. It follows that the coefficients of $\frac{1}{z^{2}}$ at both sides are equal for any $c_{2}$. However, we note that if $c_{2}=0$, then $c_{3}=0$ and inductively all $c_{n}=0$. This means $\varphi$ is constantly equal to 0 , which can not happen by the assumption. So we know $c_{2} \neq 0$. Next we check the coefficients of $\frac{1}{z^{3}}$ on both sides and obtain

$$
\frac{2 b c_{2}+c_{3}}{a^{3}}=\frac{c_{3}}{a^{2}}
$$

Thus,

$$
\frac{b}{a-1}=\frac{c_{3}}{2 c_{2}}
$$

for every non-identity element $g(z)=a z+b \in G$. Therefore, all of them share the second fixed point $-\frac{b}{a-1}$. So we have proved that in this subcase all hyperbolic elements share both fixed points.

\section{Proof of Theorem 2}

Through conjugation by an element in $\operatorname{PSL}(2, \mathbb{R})$, the proof of Theorem 2 is reduced to proving the following two propositions.

Proposition 3.1. Let $G$ be a non-discrete subgroup of $P S L(2, \mathbb{R})$ containing only hyperbolic elements with two common fixed points. Then the length spectrum $d_{L}$ defines only a pseudometric on $T(G)$.

Proof. Using the definition of $d_{L}$, one can easily verify that that $d_{L}$ is symmetric and satisfies the triangle inequality. The main work is to show that $d_{L}$ fails to distinguish all points of $T(G)$; that is, there are two distinct points whose $d_{L}$ distance is zero.

Let us use the same notation introduced in the proof of Proposition 2.2. That is, without loss of generality, we continue to assume that $G$ is a non-discrete subgroup of $\operatorname{PSL}(2, \mathbb{R})$ containing only hyperbolic elements with fixed points at 0 and $\infty$. For each $f_{\beta} \in T(G)$, we are now interested in finding the representative $f^{\beta}$ of $\left[f_{\beta}\right]$ preserving $\mathbb{H}$, where $|\beta|<1$. Let $p: \mathbb{C} \rightarrow \mathbb{C} \backslash\{0\}$ be the universal covering map and let $\tilde{f}_{\beta}: \mathbb{C} \rightarrow \mathbb{C}$ be the lift of $f_{\beta}: \mathbb{C} \backslash\{0\} \rightarrow \mathbb{C} \backslash\{0\}$ fixing 0 . We know that the strip $B=\{\zeta: 0<\operatorname{Im}(\zeta)<\pi\}$ is mapped by $p$ onto $\mathbb{H}$ and

$$
\left(\left.\tilde{f}_{\beta}\right|_{B}\right)(\zeta)=\zeta+\beta \bar{\zeta}
$$

where $\zeta=\eta+\xi i$. It is clear that $\tilde{f}_{\beta}$ maps $B$ onto a strip $C$ bounded by two parallel lines. One of them passes through the origin and $\tilde{f}_{\beta}(1)=1+\beta$ and the other passes through $\tilde{f}_{\beta}(\pi i)=(1-\beta) \pi i$. Through a rotation and a stretch, one can see that the function

$$
\tilde{h}(z)=\frac{z}{(1+\beta) \operatorname{Im}\left(\frac{1-\beta}{1+\beta} i\right)}
$$

maps the strip $C$ to the strip $\{z: 0<\operatorname{Im}(z)<\pi\}$. Since

$$
\operatorname{Im}\left(\frac{1-\beta}{1+\beta} i\right)=\operatorname{Re}\left(\frac{1-\beta}{1+\beta}\right)=\frac{1}{2}\left(\frac{1-\beta}{1+\beta}+\frac{1-\bar{\beta}}{1+\bar{\beta}}\right)=\frac{1-|\beta|^{2}}{(1+\beta)(1+\bar{\beta})},
$$

it follows that

$$
\tilde{h}(z)=\frac{1+\bar{\beta}}{1-|\beta|^{2}} z
$$


Hence the mapping $f^{\beta}$ (preserving $\mathbb{H}$ ) is given by

$$
f^{\beta}(z)=e^{\left(\tilde{h} \circ \tilde{f}_{\beta}\right)(\log z)}=z^{\frac{1+\bar{\beta}}{1-|\beta|^{2}}} \bar{z}^{\frac{\beta(1+\bar{\beta})}{1-|\beta|^{2}}},
$$

where the branch of logarithm is given by

$$
\log z=\log |z|+i \arg z \text { for }-\pi<\arg z<\pi .
$$

We know that for any $g_{t_{k}} \in G$, there exists $\lambda_{t_{k}} \in \mathbb{R}$ such that for any $z \in \mathbb{H}$, we obtain

$$
\left(f^{\beta} \circ g_{t_{k}} \circ\left(f^{\beta}\right)^{-1}\right)(z)=\lambda_{t_{k}} z .
$$

Since $f^{\beta}$ fixes the point 1 , we must have $f^{\beta}\left(e^{t_{k}}\right)=\lambda_{t_{k}}$. That is,

$$
\lambda_{t_{k}}=f^{\beta}\left(e^{t_{k}}\right)=\left(e^{t_{k}}\right)^{\frac{(1+\beta)(1+\bar{\beta})}{1-|\beta|^{2}}} .
$$

It follows that the length spectrum is given by

$$
\begin{aligned}
d_{L}\left(\left[f^{\beta_{1}}\right],\left[f^{\beta_{2}}\right]\right) & =\log \sup _{g_{t_{k}} \in G}\left\{\left|\frac{\log \tau\left(f^{\beta_{1}} \circ g_{t_{k}} \circ\left(f^{\beta_{1}}\right)^{-1}\right)}{\log \tau\left(f^{\beta_{2}} \circ g_{t_{k}} \circ\left(f^{\beta_{2}}\right)^{-1}\right)}\right|^{ \pm 1}\right\} \\
& =\log \max \left\{\left|\frac{\left(1+\beta_{1}\right)\left(1+\bar{\beta}_{1}\right)}{1-\left|\beta_{1}\right|^{2}} \frac{1-\left|\beta_{2}\right|^{2}}{\left(1+\beta_{2}\right)\left(1+\bar{\beta}_{2}\right)}\right|^{ \pm 1}\right\} .
\end{aligned}
$$

Thus, $d_{L}\left(\left[f^{\beta_{1}}\right],\left[f^{\beta_{2}}\right]\right)=0$ if and only if

$$
\frac{1-\left|\beta_{1}\right|^{2}}{\left|1+\beta_{1}\right|^{2}}=\frac{1-\left|\beta_{2}\right|^{2}}{\left|1+\beta_{2}\right|^{2}} .
$$

Using the expression (3.1) of $f^{\beta}$, one can see that this condition is equivalent to saying that $f^{\beta_{1}}$ and $f^{\beta_{2}}$ agree on the positive real axis.

The third part of Theorem 1 states that $T(G)$ is conformally identified with the open unit disk. Given a point $\beta_{2}$ with $\left|\beta_{2}\right|<1$, let $c=\frac{1-\left|\beta_{2}\right|^{2}}{\left|1+\beta_{2}\right|^{2}}$. Then the equation $\frac{1-\left|\beta_{1}\right|^{2}}{\left|1+\beta_{1}\right|^{2}}=c$ represents a horocylce, which is contained in the closed unit disk and tangent to the unit circle at -1 . Then the length spectrum $d_{L}$ is equal to 0 at any two points on the horocycle except -1 . Therefore, $d_{L}$ does not distinguish all points in $T(G)$ and hence it is just a pseudometric on $T(G)$.

Proposition 3.2. Let $G$ be as the same as given in Proposition 3.1. The Teichmüller metric on $T(G)$ corresponds to the hyperbolic metric on the open unit disk $\mathbb{D}$, and $d_{L}$ agrees with $d_{T}$ on the slice of $T(G)$ that is identified in Proposition 3.1 with the real diameter of $\mathbb{D}$.

Proof. Given any $\beta \in \mathbb{D}$, note that the expression (3.1) of $f^{\beta}$ is an extremal representative of $\left[f^{\beta}\right]$. Furthermore, for any two points $\beta_{1}$ and $\beta_{2} \in \mathbb{D}$, the work to derive the expression (3.1) for $f^{\beta}$ also shows that $f^{\beta_{1}} \circ\left(f^{\beta_{2}}\right)^{-1}$ is an extremal representative of $\left[f^{\beta_{1}} \circ\left(f^{\beta_{2}}\right)^{-1}\right]$. Note that the Beltrami coefficient of $f^{\beta}$ is given by $\beta \frac{z}{\bar{z}}$. By applying the chain rule to $f^{\beta_{1}} \circ\left(f^{\beta_{2}}\right)^{-1}$, we can conclude that the Teichmüller distance $d_{T}\left(\left[f^{\beta_{1}}\right],\left[f^{\beta_{2}}\right]\right)$ is equal to the hyperbolic distance between $\beta_{1}$ and $\beta_{2}$ on $\mathbb{D}$.

Now let $\beta_{1}=t$ and $\beta_{2}=s$ be two points on the real diameter of $\mathbb{D}$. It remains to show that $d_{L}\left(\left[f^{t}\right],\left[f^{s}\right]\right)=0$ implies that $t=s$. 
From the expression (3.2), we know

$$
d_{L}\left(\left[f^{t}\right],\left[f^{s}\right]\right)=\log \max \left\{\left(\frac{1+t}{1-t} \frac{1-s}{1+s}\right)^{ \pm 1}\right\}=\log \max \left\{\left(\frac{1+t-s-t s}{1-t+s-t s}\right)^{ \pm 1}\right\} .
$$

Since the complex dilatation of $f^{t} \circ\left(f^{s}\right)^{-1}$ has norm $|t-s| /|1-t s|$, it follows that

$$
\begin{aligned}
d_{T}\left(\left[f^{t}\right],\left[f^{s}\right]\right) \leq K\left(f^{t} \circ\left(f^{s}\right)^{-1}\right) & =\log \frac{1+|t-s| /|1-t s|}{1-|t-s| /|1-t s|} \\
& =\log \max \left(\frac{1-t s+t-s}{1-t s-t+s}\right)^{ \pm 1} \\
& =d_{L}\left(\left[f^{t}\right],\left[f^{s}\right]\right) .
\end{aligned}
$$

On the other hand, for any $g \in G$, let

$$
h_{t}=f^{t} \circ g \circ\left(f^{t}\right)^{-1} \text { and } h_{s}=f^{s} \circ g \circ\left(f^{s}\right)^{-1} \text {. }
$$

Then $f^{t}$ and $f^{s}$ induce two quasiconformal mappings between annuli, denoted by

$$
\tilde{f}^{t}: \mathbb{H} /\langle g\rangle \rightarrow \mathbb{H} /\left\langle h_{t}\right\rangle \quad \text { and } \quad \tilde{f}^{s}: \mathbb{H} /\langle g\rangle \rightarrow \mathbb{H} /\left\langle h_{s}\right\rangle .
$$

Clearly,

$$
K\left(f^{t}\right)=K\left(\tilde{f}^{t}\right) \quad \text { and } \quad K\left(f^{s}\right)=K\left(\tilde{f}^{s}\right) .
$$

Applying Wolpert's lemma [1] to the map

$$
\tilde{f}^{t} \circ\left(\tilde{f}^{s}\right)^{-1}: \mathbb{H} /\left\langle h_{s}\right\rangle \rightarrow \mathbb{H} /\left\langle h_{t}\right\rangle,
$$

we obtain

It follows that

$$
\frac{\log \tau\left(h_{t}\right)}{K\left(f^{t} \circ\left(f^{s}\right)^{-1}\right)} \leq \log \tau\left(h_{s}\right) \leq K\left(f^{t} \circ\left(f^{s}\right)^{-1}\right)
$$

$$
d_{L}\left(\left[f^{t}\right],\left[f^{s}\right]\right) \leq d_{T}\left(\left[f^{t}\right],\left[f^{s}\right]\right) .
$$

Combining the inequalities (3.3) and (3.4), we conclude that the length spectrum $d_{L}$ is a metric coinciding with $d_{T}$ on the slice of $T(G)$ that is identified with the real diameter of $\mathbb{D}$.

Propositions 3.1 and 3.2 imply our Theorem 2.

\section{References}

[1] Aвiкоff, W.: The real analytic theory of Teichmüller space. - Lecture Notes in Math. 820, Springer-Verlag, Berlin-Heidelberg-New York, 1980.

[2] Beardon, A. F.: The geometry of discrete groups. - Grad. Texts in Math. 91, Springer-Verlag, Berlin-Heidelberg-New York, 1983.

[3] Hu, J., and F. G. Jimenez-Lopez: Modified length spectrum metric on the Teichmüller space of a Riemann surface with boundary. - Ann. Acad. Sci. Fenn. Math. 39, 2014, 513-526.

[4] Lehto, O.: Univalent functions and Teichmüller spaces. - Grad. Texts in Math. 109, SpringerVerlag, New York, 1987.

[5] LI, Z.: Teichmüller metric and length spectrum of Riemann surface. - Scientia Sinica Ser. A $24,1986,265-274$.

[6] LI, Z.: Length spectrum of Riemann surfaces and Teichmüller metric. - Bull. London Math. Soc. 35, 2003, 247-254.

[7] LiU, L.: On the length spectrums of non-compact Riemann surfaces. - Ann. Acad. Sci. Fenn. Math. 24, 1999, 11-22. 
[8] Shiga, H.: On a distance defined by the length spectrum on Teichmüller space. - Ann. Acad. Sci. Fenn. Math. 28, 2003, 315-326.

[9] Sorvali, T.: On Teichmüller spaces of tori. - Ann. Acad. Sci. Fenn. Ser. A I Math. 1, 1975, $7-11$.

[10] Strebel, K.: Eine Abschätzung der Länge gewisser Kurven bei quasikonformer Abbildung. Ann. Acad. Sci. Fenn. Ser. A I Math. 243, 1957, 1-10.

[11] Strebel, K.: Zur Frage der Eindeutigkeit extremaler quasikonformer Abbildungen des Einheitskreises. - Comment. Math. Helv. 36, 1962, 306-323.

Received 1 December 2020 • Accepted 7 June 2021 • Published online 9 February 2022

Jun $\mathrm{Hu}$

Brooklyn College of CUNY

Department of Mathematics

Brooklyn, NY 11210, U.S.A.

and Graduate Center of CUNY

Ph.D. Program in Mathematics

365 Fifth Avenue, New York, NY 10016, U.S.A.

and Institute of Mathematical Sciences at NYU

Shanghai, NYU-ECNU, 3663 Zhongshan

Road North, Shanghai, 200062, P. R. China

junhu@brooklyn.cuny.edu or JHu1@gc.cuny.edu
Francisco G. Jimenez-Lopez

Universidad Autónoma de Querétaro

Facultad de Ingeniería

Cerro de las Campanas s/n, 76010 Santiago de Querétaro, Querétaro, Mexico

fjimenez@uaq.mx 